\title{
Occurrence of Fusarium Head Blight and Mycotoxins as well as Morphological Identification of Fusarium Species in Winter Wheat in Kosovo
}

\author{
V. Shala-Mayrhofer ${ }^{1}$, R. MarJaKaj ${ }^{2}$, E. VARGA ${ }^{3}$, F. Berthiller ${ }^{3}$, \\ A. Musollit ${ }^{4}$ and M. Lemmens ${ }^{4 *}$ \\ ${ }^{1}$ Division of Agronomy, Department of Crop Sciences, University of Natural Resources and Life Sciences, \\ Vienna, Konrad Lorenz Str. 24, 3430 Tulln, Austria \\ ${ }^{2}$ Ministry of Agriculture, Forestry and Rural Development, Prishtinë, Kosovo \\ ${ }^{3}$ Center for Analytical Chemistry and Christian Doppler Laboratory for Mycotoxin Metabolism, \\ Department for Agrobiotechnology, University of Natural Resources and Life Sciences, \\ Vienna, Konrad Lorenz Str. 20, 3430 Tulln, Austria \\ ${ }^{4}$ Institute for Biotechnology in Plant Production, Department for Agrobiotechnology, \\ University of Natural Resources and Life Sciences, Vienna, Konrad Lorenz Str. 20, 3430 Tulln, Austria
}

(Received 29 June 2014; Accepted 15 October 2014;

Communicated by Á. Mesterházy and X.F. Zhang)

Wheat (Triticum aestivum L.) is an important cereal crop in Kosovo and a major component of population food. Fusarium head blight (FHB) of wheat is an economically very significant disease. FHB leads to various losses in quality like reduced germination of seeds, reduced baking quality and reduced nutritional quality through mycotoxin contamination. In 2010 and 2011 the incidence and identity of the Fusarium spp. infecting wheat in Kosovo as well as mycotoxin contamination was investigated. The results of two years research work show that the incidence of FHB on winter wheat in 2010 was low $(<6 \%)$. In the year 2011 the disease incidence was clearly higher (up to $31 \%$ ). Based on morphological characters, F. graminearum was the most frequently Fusarium sp. identified on wheat kernels in the year $2010(100 \%)$ and $2011(98 \%)$. Less frequently isolated species included F. cerealis $(<1 \%)$ and $F$. avenaceum $(<1 \%)$. Wheat flour samples were analyzed by liquid chromatography coupled to mass spectrometry and found to be contaminated with a variety of mycotoxins, most importantly deoxynivalenol and zearalenone. This is the first report on the incidence as well as on the identification of Fusarium species isolated from naturally infected winter wheat in Kosovo.

Keywords: Fusarium head blight, Fusarium species, Triticum aestivum, Kosovo

\section{Introduction}

In Kosovo approximately $80000-100000$ ha of wheat are cultivated annually (Statistical Office of Kosovo 2009). The seeds for cultivation are largely imported from neighbouring countries. To date no cultivars for multiplication are available. Also, the average

\footnotetext{
* Corresponding author; E-mail: marc.lemmens@boku.ac.at
} 
wheat yield of 2,500-3,000 $\mathrm{kg} \mathrm{ha}^{-1}$ (Statistical Office of Kosovo 2009) does not fulfil the domestic demand of Kosovo. As a consequence the selection of suitable wheat cultivars for the producing region Kosovo is crucial in order to increase crop yield and to improve quality. In addition to the parameters "quality and yield", fungal diseases represent a central problem.

Fusarium head blight (FHB) in wheat is a widespread disease around the world including all European cereal-growing areas (Bottalico and Perrone 2002). The extent of the damage increases with the intensification of grain production. The disease leads to various losses in quality, including reduced germination in seeds, reduced baking quality due to the breakdown of wheat storage proteins and reduced nutritional quality through contamination with mycotoxins (Champeil et al. 2004; Mesterházy 2005). Based on economic and environmental aspects, host plant resistance is considered to be the most appropriate method to control FHB (Ruckenbauer et al. 2001).

In extreme cases the wheat heads infected with Fusarium are completely bleached (white heads) and show a limited kernel set. The kernels are often shrivelled. Usually individual spikelets or parts of the wheat head are infected. The infected parts die off and appear somewhat lighter (straw like colour). Often, a salmon-coloured coating (spore agglomeration) also occurs on the chaff. A white, rose or red colouring of the grains can also be a consequence of this disease. If the infection of the wheat takes place in a very early phase during flowering, already the young, green wheat heads can be strongly attacked by fungi. The fungus is seed-borne or initiates from the soil, however, the most important source of infection is represented by crop debris from the previous crop on the soil surface on which the fungi prevail over winter (Sutton 1982). Maize as a preceding crop promotes the incidence of Fusarium spp. including F. graminearum in winter wheat (Dill-Macky and Jones 2000; Schaafsma et al. 2005; Blandino et al. 2010; Vogelgsang et al. 2011). An explanation for this fact is the infection progress of the pathogen. Already at the time of maize maturation onset, Fusarium can penetrate the lower stem portion of the maize plant. After the harvest, most of the maize stubble is colonized with Fusarium. The maize straw remaining on the soil, with its high nitrogen content, appears to be a suitable culture medium for the cultivation of the main progeny form of the fungi in the next vegetation period. The ascospores produced by Gibberella zeae in the perithecia can be actively ejected and are the source of the new infection. This takes place in a monocyclic occurrence from the spore repositories on the soil surface directly onto the wheat heads. The level of infection highly depends on the year and location and is also largely influenced by weather conditions (Lemmens et al. 2004; Lori et al. 2009; Lakhesar et al. 2010).

Fusarium head blight in wheat is caused by several species of Fusarium. In general, the causal agents of FHB in Europe are primarily F. graminearum (teleomorph Gibberella zeae), F. culmorum (teleomorph unknown) and $F$. avenaceum (teleomorph G. avenacea). Less frequently represented species are $F$. poae, F. cerealis, F. equiseti, F. sporotrichioides, $F$. tricinctum and, to an even lesser extent also $F$. acuminatum, $F$. subglutinans, $F$. solani, $F$. oxysporum, $F$. verticillioides, $F$. semitectum and $F$. proliferatum (Bottalico and Perrone 2002; Lemmens et al. 2004; Stepieň et al. 2008). 
The aim of this study was 1) to demonstrate the possible role of Fusarium head blight of wheat in Kosovo, 2) to identify the Fusarium species isolated from naturally infected wheat kernels, and 3) to demonstrate that the mycotoxins predicted to be produced by these Fusarium spp. were indeed present in kernels samples. Within the framework of this project, field and laboratory tests were carried out on wheat in 2010 and 2011 at five locations from the two most important wheat growing regions in Kosovo (the Kosovo region and the Dukagjini region).

\section{Materials and Methods}

\section{Locations and cultivars}

During the vegetative period in 2010 and in 2011 five locations within two regions of Kosovo were selected (Fig. 1). The first region was the Kosovo region with the locations Baks and Skifter: altitude around 550 meters, mean annual temperature $16.5^{\circ} \mathrm{C}$, soil type: vertisol and chernozem. The second region was the Dukagjini region (Gurakoc, Novosellë, Prizren): altitude around 370 meters, mean annual temperature $17.5^{\circ} \mathrm{C}$, soil type:

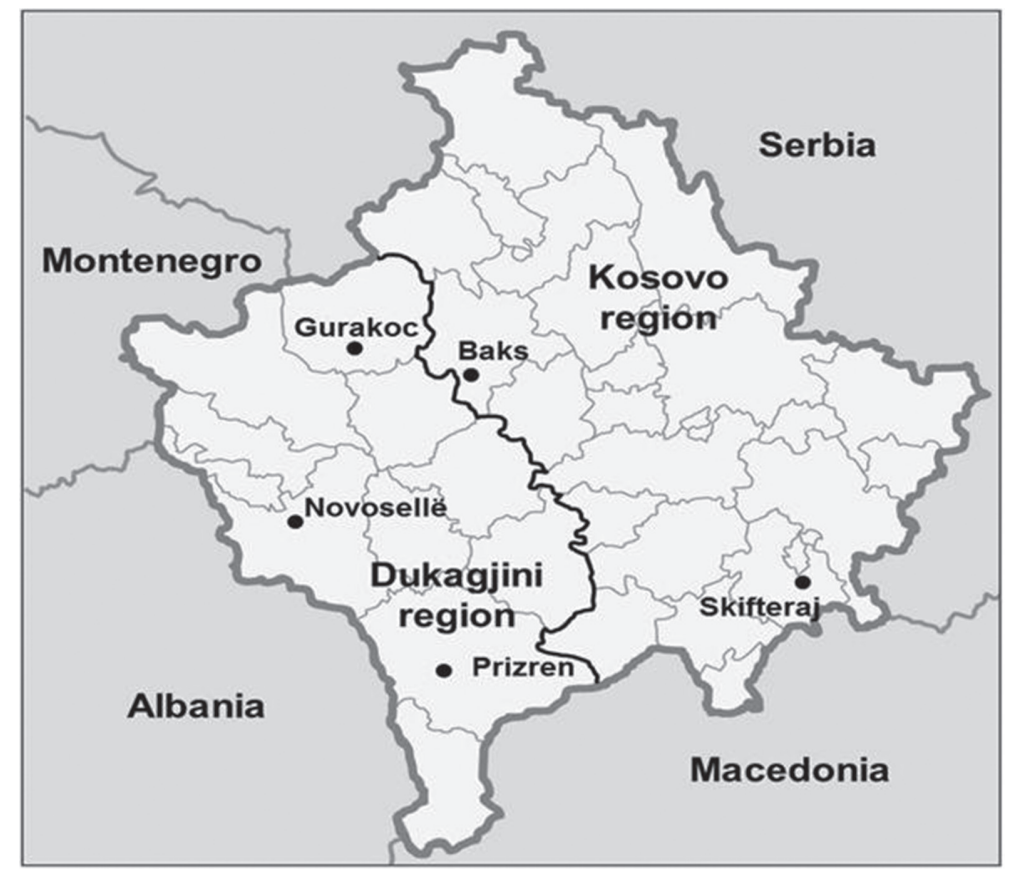

Figure 1. Map of Kosovo showing the locations where visual field analyses and Fusarium-infected kernels were collected in 2010 and 2011 (map modified after Nord Nord West Version of 23 November 2009). http://commons.wikimedia.org/wiki/File:Kosovo_location_map.svg?uselang=en (Accessed 15 December 2012) published under the licence of Creative CommonsAttribution-ShareAlike 3.0 Unported (CC BY-SA 3.0), http://creativecommons.org/licenses/by-sa/3.0/deed.en 
alluvial, brown, alluvium/pseudogley and pelosol, continental climate. The preceding crop was maize in all cases. The fields were selected from local farmers and were located in the main wheat growing regions in Kosovo. The sampled wheat cultivars depending on the location were Evropa, Renesanca and Ritma. For these genotypes there is no information available on the resistance to Fusarium head blight. Evropa was sampled at the locations Prizren and Skifter in 2010 and 2011, at Baks and at Gurakoc in 2011. Renesanca was investigated at Novosellë in 2010 and 2011 and at Gurakoc in 2010. Ritma was sampled only at the location Baks in 2010. The areas of the plots were 0.75 ha in Baks, 0.4 ha in Skifter, 0.4 ha in Novosellë, 0.3 ha in Gurakoc and 0.7 ha at the location Prizren. The number of replications was four. As usual in Kosovo, fertilization was done with N:P:K 120:80:80 $\mathrm{kg} \mathrm{ha}^{-1}$. To control weeds the following herbicides were used depending on the location: Senkor, Agrosan, Mustang and Maton 2.4-D. The rates were applied according to the protocol of the producers. No fungicides were applied.

\section{Field analyses}

For disease assessment wheat heads were rated at the hard dough stage. One hundred wheat heads from each replication were harvested by hand and subsequently analyzed in the laboratory for disease incidence (DI; percentage of diseased ears) and disease severity (DS) on a 0 to 5 scale in which 0 equals no symptoms, $1=1-5 \%, 2=6-10 \%, 3=11-$ $25 \%, 4=26-50 \%$ and 5 equals infection of over $50 \%$ of spikelets of the wheat ear (EPPO 1997).

\section{Laboratory analysis}

To isolate Fusarium species, kernels with visible symptoms were collected directly during the visual analysis from the wheat ears. They were surface-sterilised in $80 \%$ ethanol for two minutes, air dried and transferred onto Synthetic Nutrient-poor Agar (SNA) ( $1 \mathrm{~g} \mathrm{KH}_{2} \mathrm{PO}_{4} ; 1 \mathrm{~g} \mathrm{KNO}_{3} ; 0.5 \mathrm{~g} \mathrm{MgSO}_{4} 7 \mathrm{H}_{2} \mathrm{O} ; 0.5 \mathrm{~g} \mathrm{KCl} ; 0.2 \mathrm{~g}$ glucose; 0.2 g saccharose; $1 \mathrm{~L} \mathrm{H}_{2} \mathrm{O}$ dest.; $22 \mathrm{~g}$ agar; $0.6 \mathrm{~mL} 1 \mathrm{~N} \mathrm{NaOH}$ ) supplemented with antibiotics (Nirenberg 1981; modified). After 7-14 days incubation in an incubator (temperature $25^{\circ} \mathrm{C}$ and humidity $80 \%$, illumination with dark UV light), a single spore technique was used to obtain pure cultures for identification. Potato dextrose agar (PDA) (Difco, Detroit, MI) medium was used to assess colony characteristics such as pigmentation and growth rate. SNA medium, supplemented with a piece of filter paper to promote sporulation, was used to examine conidial morphology and to detect the presence of chlamydospores. Black UV light $(36 \mathrm{~W})$ was used to improve sporulation of isolates. Individual Fusarium species were identified (Nirenberg 1981; Nelson et al. 1983; Singh et al. 1991). From the 250 kernels we collected in total during both years, 228 kernels were infected with Fusarium species. From the other kernels no Fusarium was growing out or we were not able to obtain pure Fusarium colonies due to contamination with other fungi. 


\section{Mycotoxins}

Detection and quantification of the mycotoxins in wheat was carried out by high performance liquid chromatography-tandem mass spectrometry. A multi-mycotoxin method for wheat (Sulyok et al. 2006) was enhanced to cover a total of 186 mycotoxins and other fungal and bacterial metabolites (Vishwanath et al. 2009). The kernel samples were taken after threshing with a plot combiner and were milled. Only samples from 2011 were analyzed (Table 2) and the 4 replications were pooled. From $1 \mathrm{~kg}$ milled wheat a representative sub-sample of $10.00 \pm 0.01 \mathrm{~g}$ ground wheat was extracted for $90 \mathrm{~min}$ on a rotary shaker using $40 \mathrm{~mL}$ acetonitrile/water/acetic acid (79/20/1, v/v/v). The centrifuged extract was diluted $1+1$ with acetonitrile/water/acetic acid $(20 / 79 / 1, v / v / v)$. Five $\mu \mathrm{L}$ of the diluted extract were injected using an Agilent 1100 HPLC system (Waldbronn, Germany). Separation was achieved on a $150 \times 4.6 \mathrm{~mm}$ i.d., $5 \mu \mathrm{m}$ particle size, Phenomenex Gemini RP-C18 column (Torrance, USA) using a linear aqueous ammonium acetate/ acetic acid/methanol gradient from 10 to $97 \%$ organic solvent at a flow rate of $1 \mathrm{~mL} / \mathrm{min}$ at $25{ }^{\circ} \mathrm{C}$. Detection of mycotoxins was achieved using an ABSciex4000 QTrap mass spectrometer (Foster City, USA) equipped with a TurboV electrospray source. Two injections were used for each sample, one for each ion source polarity. The scheduled single reaction monitoring mode was used to acquire data, which were further evaluated using Analyst version 1.5.2. Mixed standard solutions containing all 186 analyses at different concentration were used for external calibration. All data were corrected for the apparent recovery according to Sulyok et al. (2006).

\section{Statistical analyses}

Data taken were analyzed for variance (ANOVA) and the means as well as the standard deviations $(n=4)$ were calculated. The analysis of variance was performed only for the disease incidence and severity. SPSS Version 15.0 for MS Windows was used for the analyses.

\section{Results}

Generally in 2010 the disease incidence (DI) was low and in all cultivars below 6\% (Table 1). In 2011 the DI was higher and varied from 4 to $31 \%$. Also, disease severity (DS) showed a similar picture: DS was higher in the second season (from 11 to $24 \%$ ). The highest DI and disease severity (DS) in both years showed the cultivar Evropa at the location Prizren.

Identified Fusarium spp. from each cultivar in 2010 and 2011 are summarized in Table 1. Based on morphological characters F. graminearum was the only species identified in 2010. Two Fusarium strains isolated from the cultivar Renescanca at the location Novosellë could not be clearly identified at the species level. In 2011, the most frequently Fusarium spp. identified was again $F$. graminearum (98\%). Less frequently isolated species included $F$. cerealis and $F$. avenaceum $(<1 \%)$. 
ShalA-MaYrhofer et al.: Fusarium and Mycotoxins on Wheat in Kosovo

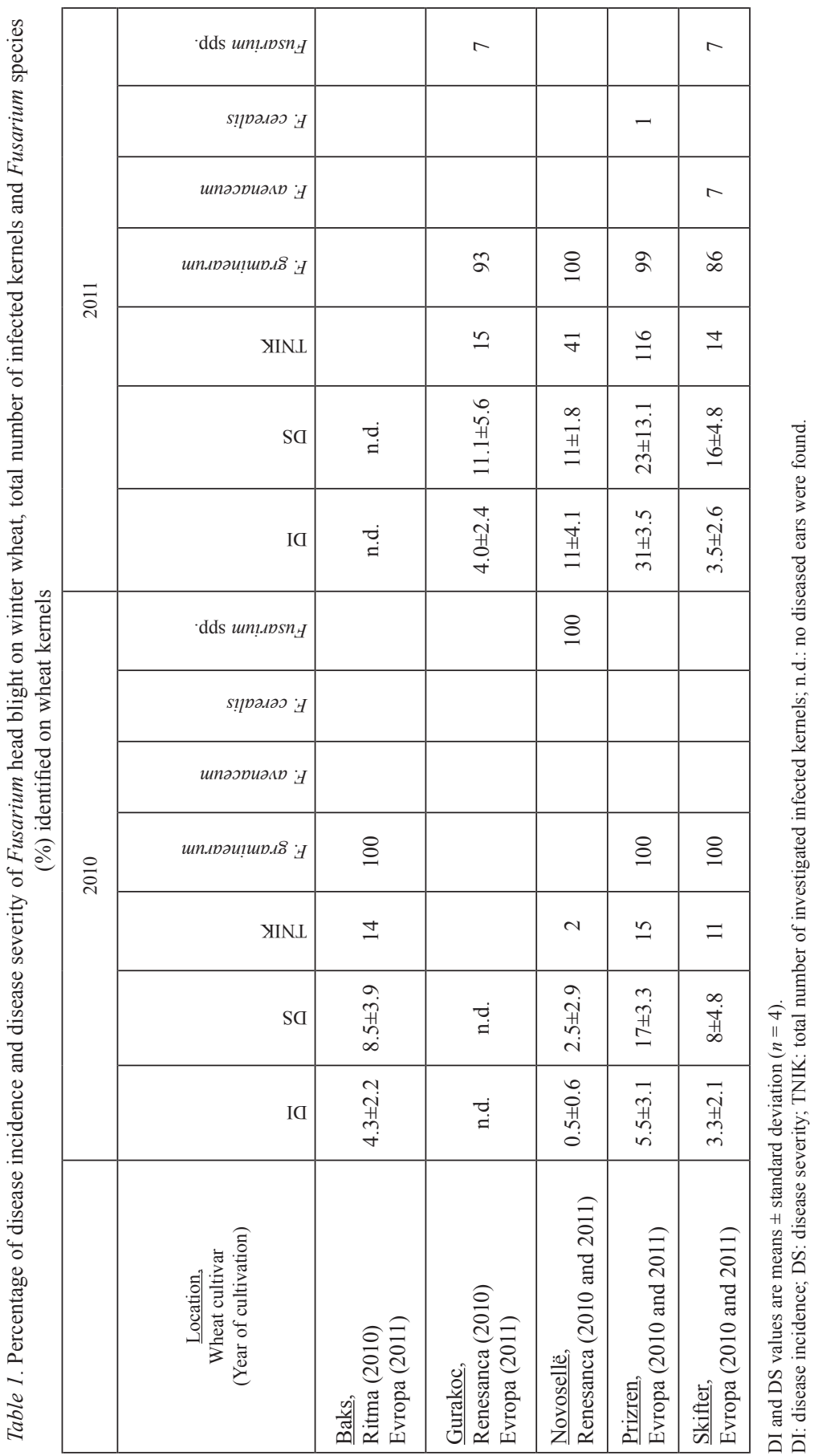

Cereal Research Communications 43, 2015 


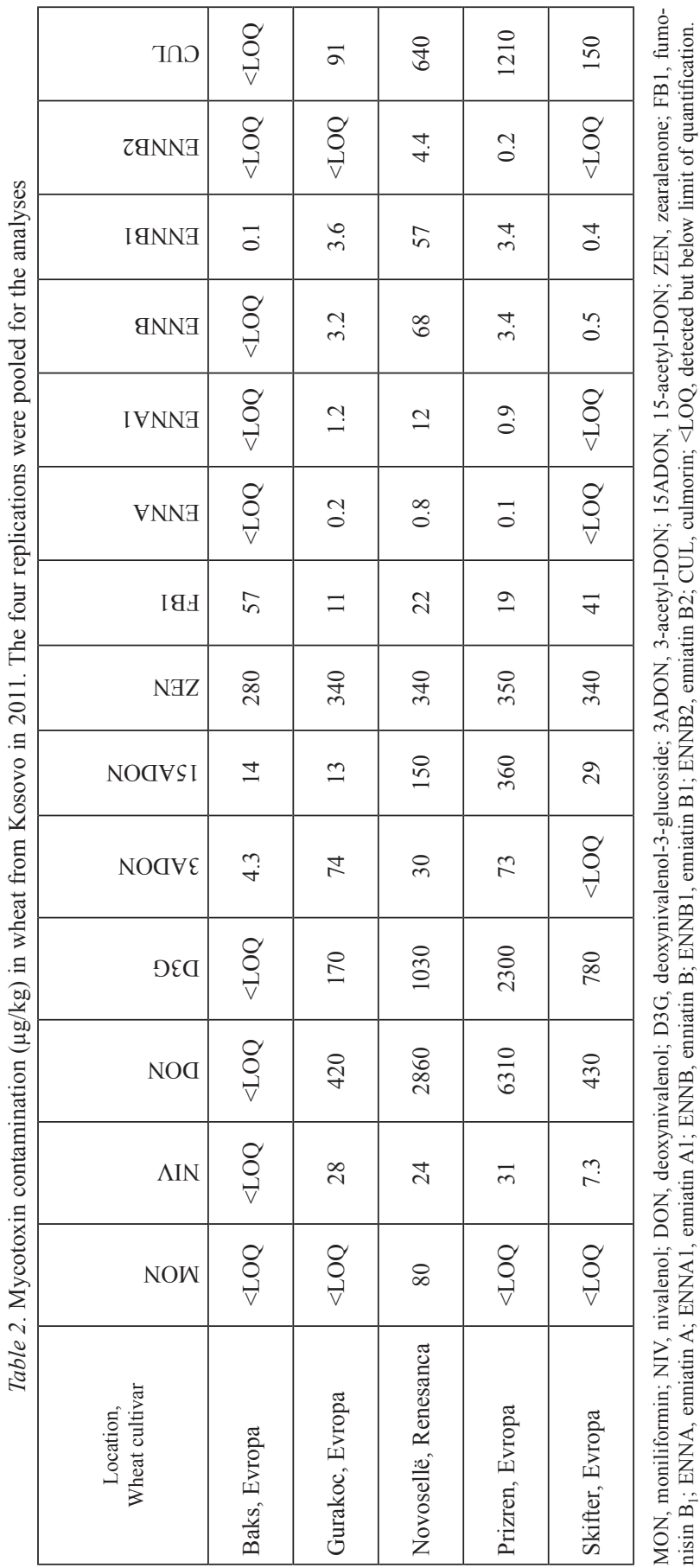

Cereal Research Communications 43, 2015 
Regarding mycotoxins, the following substances were detected in the wheat samples from 2011 (Table 2): moniliformin (MON), nivalenol (NIV), deoxynivalenol (DON), deoxynivalenol-3-glucoside (D3G), 3-acetyl-DON (3ADON), 15-acetyl-DON (15ADON), zearalenone (ZEN), fumonisin $\mathrm{B}_{1}$ (FB1), enniatin A (ENNA), enniatin A1 (ENNA1), enniatin B (ENNB), enniatin B1 (ENNB1), enniatin B2 (ENNB2) and culmorin (CUL), all of which are produced by various Fusarium spp. As expected from the high DI and DS, the sample obtained from Prizren was also the one with the highest concentration of mycotoxins, exceeding $6 \mathrm{mg} / \mathrm{kg}$ DON, $2 \mathrm{mg} / \mathrm{kg}$ D3G and $1 \mathrm{mg} / \mathrm{kg}$ CUL. Interestingly, the level of ZEN found in the samples was very constant (at around $300 \mu \mathrm{g} / \mathrm{kg}$ ), regardless of the location. Enniatins were found in all samples at very low concentrations, with the exception of the wheat from Novosellë, which was contaminated with ENNB and ENNB1 at levels exceeding $50 \mu \mathrm{g} / \mathrm{kg}$. In the sample from Baks, which showed no FHB symptoms in planta, with the exception of ZEN, no other mycotoxins could be found in relevant concentrations.

\section{Discussion}

The purpose of this study was to analyze the incidence and identity of the Fusarium species isolated from naturally infected winter wheat kernels in Kosovo as well as mycotoxin contamination. In particular, through relevant field analyses and laboratory tests, this two-year research work generated important information about the Fusarium spp. encountered in Kosovo on naturally infected wheat and their contamination with mycotoxins.

The results showed that Fusarium head blight on wheat is an important problem in Kosovo. The disease incidence of FHB on wheat on the sampled fields varied in both years: in 2010 it was low, but in 2011 the disease incidence and severity was substantially higher. In one location $31 \%$ of the investigated ears showed symptoms. On the other hand, the disease was not detected on one location in each season. It is very well known that the level of incidence of FHB is highly depending on the year, location, cultivar and weather conditions (Dill-Macky and Jones 2000; Champeil et al. 2004; Lori et al. 2009). The preceding crop in all locations was maize, which promotes the incidence of Fusarium spp. in winter wheat (Vogelgsang et al. 2011). The highest percentage of DI and DS of FHB was recorded at the location Prizren, exactly the same place where we have also seen the highest DI of Fusarium ear rot in the preceding maize crop (Shala-Mayrhofer et al. 2013). It has to be mentioned, that at this location irrigation was used during maize cultivation. We hypothesize that irrigation resulting in a high ear rot disease level in maize is responsible for a high inoculum potential in the following wheat crop.

Based on morphological characters, $F$. graminearum was the most frequent species identified. Less frequently isolated species included $F$. cerealis and $F$. avenaceum. The latter were found only in 2011. These toxigenic Fusarium species isolated from wheat are in good accordance with previous reports in other European countries (Mesterházy 2005; Bottalico and Perrone 2002) as well as in Serbia (Lević et al. 2009) and in Croatia (Ivič et al. 2009; Spanič et al. 2010). 
Mycotoxins are secondary metabolic products of various fungi, which play an important role in the spoiling of animal feed and human food products, and represent one of the most serious consequences of Fusarium infection. While the overall sample set was very small, still high amounts of toxins were found in the sample from Prizren and Novosellë. The values found for DON in those two samples are exceeding the regulated maximum level $(1250 \mu \mathrm{g} / \mathrm{kg}$ ) for human consumption in the EU (Commission Regulation (EC) No. 1881/2006). F. graminearum and F. cerealis can also produce NIV and ZEN. All investigated wheat samples would exceed the European Union regulated limit for ZEN (100 $\mu \mathrm{g} /$ $\mathrm{kg}$ ) for unprocessed cereals intended for food. We have no explanation for this high level of ZEN and it should be stated that a larger set of samples should be investigated to confirm this. All other detected toxins are either not regulated in the EU or well below the maximum level in case of FB1. Interestingly, culmorin (Ghebremeskel and Langseth 2001 ) and hydroxyculmorins (not shown) - secondary metabolites from $F$. graminearum or F. culmorum - were found in the majority of the samples. The toxicity of culmorin is rather low, therefore the found values are of little concern. F. graminearum probably causes the contamination with the monoacetyl-deoxynivalenols. F. avenaceum could be responsible for the MON and ENN contamination of the kernels. Fumonisins are toxins known to be produced by $F$. proliferatum and $F$. verticillioides which are important Fusarium ear rot pathogens in maize. They are considered to be less pathogenic or opportunistic Fusarium species on small grain cereals (Bottalico and Perrone 2002). Last but not least the fraction "masked" trichothecenes (deoxynivalenol-3-glucoside) should be mentioned. D3G is a plant metabolite of DON. The D3G concentrations in 3 out of 4 samples were in the range of $36-40 \%$ of the non-conjugated mycotoxin, but exceeds the contamination with DON in the sample from Skifter.

In conclusion we found that Fusarium head blight on wheat in Kosovo is present and the incidence can be high. The most frequently Fusarium sp. identified on wheat kernels was F. graminearum. Wheat samples were contaminated with up to 14 different Fusarium mycotoxins including D3G. Based on these results, further research should be done in Kosovo and the mycotoxins deoxynivalenol and zearalenone should be monitored to control food and feed quality. Furthermore, suitable wheat varieties should be selected for the producing region in Kosovo in order to increase FHB resistance and crop yield.

\section{Acknowledgements}

The work presented in this paper is part of the project "Cooperation in academic, scientific and professional fields between the Faculty of Agriculture and Veterinary in Prishtina, Kosovo and the University of Natural Resources and Life Sciences, Vienna, Austria" which is supported by the Austrian Development Agency (ADA). Furthermore, we want to thank the Federal Ministry of Economy, Family and Youth, the National Foundation for Research, Technology and Development, BIOMIN Holding GmbH and Nestec Ltd for funding the Christian Doppler Laboratory for Mycotoxin Metabolism. 


\section{References}

Blandino, M., Pilati, A., Reyneri, A., Scudellari, D. 2010. Effect of maize crop residue density on Fusarium head blight and on deoxynivalenol contamination of common wheat grains. Cereal Res. Commun. 38:550 559.

Bottalico, A., Perrone, G. 2002. Toxigenic Fusarium species and mycotoxins associated with head blight in small-grain cereals in Europe. Eur. J. Plant Pathol. 108:611-624.

Champeil, A., Dore, T., Fourbet, J.F. 2004. Fusarium head blight: epidemiological origin of the effects of cultural practices on head blight attacks and the production of mycotoxins by Fusarium in wheat grains. Plant Sci. 166:1389-1415.

Commission Regulation (EC) No 1881/2006 of 19 December 2006 setting maximum levels for certain contaminants in foodstuffs. Official J. of the European Union L 364:5-24.

Dill-Macky, R., Jones, R.K. 2000. The effect of previous crop residues and tillage on Fusariumhead blight of wheat. Plant Dis. 84:71-76.

EPPO, European and Mediterranean Plant Protection Organisation. 1997. Paris, France. Guidelines for the efficacy evaluation of plant protection products. Fungicides and Bactericides 2:33-44.

Ghebremeskel, M., Langseth, W. 2001. The occurrence of culmorin and hydroxy-culmorins in cereals. Mycopathologia 152:103-108.

Ivič, D., Domijan, A.M., Perajca, M., Milicevič, T., Cvetkovič, B. 2009. Fusariumspp. contamination of wheat, maize, soybean, and pea grain in Croatia. Arhiv Higijenu Radai Toksikolgiju 60:435-442.

Lakhesar, D.P.S., Backhouse, D., Kristiansen, P. 2010. Accounting for periods of wetness in displacement of Fusarium pseudograminearum from cereal straw. Ann. Appl. Biol. 157:91-98.

Lemmens, M., Bürstmayr, H., Krska, R., Schumacher, R., Grausgruber, H., Ruckenbauer, P. 2004. The effect of inoculation treatment and long-term application of moisture on Fusarium head blight symptoms and deoxynivalenol contamination in wheat grains. Eur. J. Plant Pathol. 110:229-308.

Lević, J.T., Stanković, S.Z., Kranjaja, V.S., Bočarov-Stančić, A.S. 2009. Fusarium species: The occurrence and the importance in agriculture of Serbia. Procs Nat. Acad. of Sci., MaticaSrpska Novi Sad 116:33-48.

Lori, G.A., Sisterna, M.N., Sarandón, S.J., Rizzo, I., Chidichimo, H. 2009. Fusarium head blight in wheat: Impact of tillage and other agronomic practices under natural infection. Crop Prod. 28:495-502.

Mesterházy, Á. 2005. Common resistance to different Fusarium spp. causing Fusarium head blight in wheat. Eur. J. Plant Pathol. 112:267-281.

Nelson, P.E., Toussoun, T.A., Marasas, W.F.O. 1983. Fusarium species: An Illustrated Manual for Identification. The Pennsylvania State University Press. University Park and London, UK.

Nirenberg, H. 1981. A simplified method for identifying Fusarium spp. occurring on wheat. Can. J. Bot. 59:1599-1609.

Ruckenbauer, P., Buerstmayr, H., Lemmens, M. 2001. Present strategies in resistance breeding against scab (Fusarium spp.). Euphytica 119:121-127.

Schaafsma, A.W., Tamburic-Illincic, L., Hooker, D.C. 2005. Effect of previous crop, tillage, field size, adjacent crop, and sampling direction on airborne propagules of Gibberella zeae/Fusarium graminearum, Fusarium head blight severity, and deoxynivalenol accumulation in winter wheat. Can. J. Plant Pathol. 27:217-224.

Shala-Mayrhofer, V., Marjakaj, R., Berthiller, F., Musolli, A., Berisha, D., Kelmendi, B., Lemmens, M. 2013. Occurrence of Fusarium ear rot, morphological identification of Fusarium species and mycotoxin contamination on maize in Kosovo. Food Additives and Contaminants Part B 6:237-243.

Singh, K., Frisvad, J.C., Marthur, S.B. 1991. An Illustrated Manual on Identification of some Seed-borne Aspergilli, Fusaria, Penicillia and their Mycotoxins. The Technical University of Denmark, Printing AiOTryk as Odense, Denmark.

Statistical Office of Kosovo (SOK). 2009. Series 2: Agriculture and Environment Statistics. Agricultural household survey 2007. Prishtina, Kosovo: Statistical Office of Kosovo, http://ask.rks-gov.net/bujqesia.

Spanić, V., Lemmens, M., Drezner, G. 2010. Morphological and molecular identification of Fusarium species associated with head blight on wheat in East Croatia. Eur. J. Plant Pathol. 128:511-516.

Stepieñ, Ł., Popiel, D., Koczyk, G., Chelkowski, J. 2008. Wheat-infecting Fusarium species in Poland-their chemotypes and frequencies revealed by PCR assay. J. Appl. Genet. 49:433-441. 
Sulyok, M., Berthiller, F., Krska, R., Schumacher, R. 2006. Development and validation of a liquid chromatography/tandem mass spectrometric method for the determination of 39 mycotoxins in wheat and maize. Rapid Commun. in Mass Spectrometry 20:2649-2659.

Sutton, J.C. 1982. Epidemiology of wheat head blight and maize ear rot caused by Fusarium graminearum. Can. J. Plant Pathol. 4:195-209.

Vishwanath, V., Sulyok, M., Labuda, R., Bicker, W., Krska, R. 2009. Simultaneous determination of 186 fungal and bacterial metabolites in indoor matrices by liquid chromatography/tandem mass spectrometry. Anal. and Bioanal. Chem. 395:1355-1372.

Vogelgsang, S., Hecker, A., Musa, T., Dorn, B., Forrer, H.D. 2011. On-farm experiments over 5 years in a grain maize/winter wheat rotation: effect of maize residue treatments on Fusarium graminearum infection and deoxynivalenol contamination in wheat. Mycotoxin Res. 27:81-96. 